\title{
Benefits of early enteral nutrition in extremely low birth weight infants
}

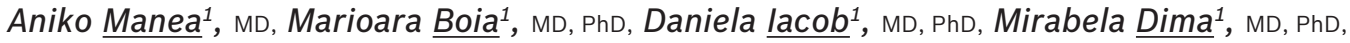 \\ Radu Emil lacob², MD, PhD
}

INTRODUCTION Extremely low birth weight (ELBW) infants (i.e. preterm infants weighing $<1,000 \mathrm{~g}$ ) often present with morphofunctional multiple organ immaturity. This study aimed to determine whether early enteral feeding improves digestive tolerance, and whether there is a difference in growth between ELBW infants who were fed with formula and those who were fed with breast milk.

METHODS This study was conducted from 2012-2013 and involved 34 ELBW infants from the Preterm Neonatology Clinic of the 'Louis Turcanu' Clinical Children's Hospital Timisoara, Romania. Early enteral nutrition was introduced for all the infants - Group I was fed with formula, while Group II was fed with breast milk. Infants in each group were given their designated type of milk (formula/breast milk), using the same feeding method and the same volume rate advancement. They were monitored for any evidence of digestive intolerance (i.e. clinical signs of infection and necrotising enterocolitis $[N E C])$. Their growth curves and signs of infection were also monitored.

RESULTS The average weight gained per week was greater among the infants in Group II than in Group I (120.83 g vs $97.27 \mathrm{~g}$ ). The incidence of infection was $100 \%$ in Group I and $66.6 \%$ in Group II. Two of the infants in Group I developed NEC.

CONCLUSION Early enteral feeding helped to improve the weight of ELBW infants. Breast milk was more effective than formula at improving the weight of these infants. Feeding with formula increased the incidence of NEC, invasive infection and morbidity among ELBW infants.

Keywords: ELBW infant, formula, human milk, necrotising enterocolitis

\section{INTRODUCTION}

Morphofunctional multiple organ immaturity in preterm infants who weigh $<1,000 \mathrm{~g}$ at birth often results in severe diseases. Preterm birth occurs at a time when the digestive, excretory and nervous systems are still immature. Although the gastrointestinal (Gl) tract is anatomically fully developed at 20 weeks of gestation, several Gl functions develop later. Therefore, preterm infants experience certain limitations in GI function. ${ }^{(1)}$ With the sudden interruption of placental transfer following the preterm birth, these infants require early nutritional intake in order to stop weight loss, compensate for metabolic deficiencies and ensure growth needs are met according to their metabolic potential. ${ }^{(2)}$ Preterm infants need nutrition to ensure that their growth and functional maturity after birth is steady and similar to that of full-term infants of the same postconceptional age. ${ }^{(3)}$ The American Academy of Pediatrics recommends that the diet of preterm infants results in a growth ratio comparable to that in the third trimester of pregnancy, without putting stress on the infants' immature metabolic and renal functions. ${ }^{(4)}$

Early enteral nutrition, also known as minimal enteral nutrition, refers to the commencement of feeding of preterm infants (using either human breast milk or formula) within the first 72 hours of life..$^{(1,5,6)}$ The benefits of early enteral nutrition are that it (a) ensures intake of active trophic substances that stimulate the development of mucosal epithelium, the intestinal immune system and microbiocenosis; (b) stimulates motility, enzyme production, Gl hormone release, the emergence of the swallowing reflex and, later, the emergence of the sucking reflex; (c) improves digestive tolerance, allows a faster increase in volume rate, helps infants achieve full enteral nutrition and ensures their nutritional needs; and (d) limits postnatal weight loss and ensures a height-weight growth similar to that of full-term infants of the same postconceptional age..$^{(1-3,5,7)}$ In fact, a delay in minimal enteral nutrition may increase the risk of necrotising enterocolitis (NEC). ${ }^{(7)}$ The administration of minimal enteral nutrition together with parenteral nutrition may help to reduce intestinal mucosal atrophy and prevent bacterial translocation..$^{(8-10)}$ Parenteral nutrition is recommended when typical nutritional and metabolic needs are not adequately met by enteral nutrition; it significantly impacts the nutritional status of the infant and reduces neonatal mortality. ${ }^{(11,12)}$ Exclusive enteral nutrition can be provided during the first 5-10 days of life. . $^{(3,5)}$

The use of human breast milk for enteral nutrition is preferred for preterm infants, as it provides adequate amounts of energy, proteins, lipids, carbohydrates, microelements, and water for growth and development. Furthermore, breast milk contains antimicrobial factors (e.g. immunoglobulin A, leucocytes, lactoferrin and lysozymes), and growth hormones and factors.

The present study aimed to: (a) determine the effects of early enteral nutrition on the improvement of digestive tolerance; (b) monitor the incidences of invasive infections and NEC in extremely low birth weight (ELBW) infants (i.e. preterm infants

${ }^{1}$ Department of Neonatology, ${ }^{2}$ Department of Pediatric Surgery, Victor Babeş University of Medicine and Pharmacy, Timișoara, Romania

Correspondence: Dr Daniela lacob, Lecturer, Department of Neonatology, Victor Babeş University of Medicine and Pharmacy, Timișoara, 2 Eftimie Murgu Square, 300041 Timişoara, Romania. danielariacob@yahoo.com 
weighing < 1,000 g); (c) assess whether formula feeding, as compared to breast milk feeding, impacts the growth and development of preterm infants; and (d) determine the optimal length of parenteral feeding and hospitalisation until the infant attains a weight of 2,500 g.

\section{METHODS}

This study was conducted in the Preterm Neonatology Clinic of the 'Louis Turcanu' Clinical Children's Hospital Timisoara, Romania, over a period of two years (2012-2013). Although 753 preterm infants were born during the study period, only 34 were enrolled in the study. Infants who were included in the study weighed $<1,000 \mathrm{~g}$ and were admitted to our clinic $\leq 48$ hours after birth. Infants who died before reaching a weight of 2,500 g, were admitted to our clinic $>48$ hours after birth or had major congenital malformations were excluded from the study.

All preterm infants received early enteral nutrition within the first 24-48 hours of life. They were divided into two groups: (a) Group I $(n=16)$, which was made up of infants who were fed with formula, and (b) Group II ( $n=18)$, which was made up of infants who were fed with human breast milk. Group I was administered formula due to one of the following reasons: (a) there was a temporary or permanent contraindication to breastfeeding $(n=9)$; $(b)$ the mother was not hospitalised together with the infant $(n=5)$; or (c) the mother suffered from agalactia $(n=2)$. In both groups, enteral nutrition was supplemented with parenteral nutrition at various periods of time, as needed. Parenteral nutrition was administered according to the fluid and caloric needs of the infants, depending on their weight and age, in order to ensure the same water and energy intake for all infants in the study group. The volume of parenteral nutrition decreased as the enteral nutrition increased.

Once the enteral nutrition was started, each group received the designated type of milk (i.e. human breast milk or formula), using the same feeding method (i.e. continuous, followed by bolus gavage), until the initiation of bottle-feeding. The rates at which the feeding volume was increased were the same for both groups. Enteral nutrition was initiated with 1-2 cc/kg of breast milk or formula. The amount of milk given was increased progressively depending on the infant's tolerance (up to 1-2 cc/kg/day along with parenteral feeding). When enteral nutrition reached $100 \mathrm{cc} / \mathrm{kg} /$ day, the infants in Group II (i.e. infants who were fed human breast milk) received additional human milk fortifiers in their feed.

All infants were monitored for digestive intolerance and clinical signs of NEC (i.e. bile-stained and blood-tinged gastric residual, abdominal bloating and melaena stool). Nonspecific clinical signs of infection (e.g. thermal instability, hypotonia, bradycardia, peripheral hypoperfusion, increase in apnoea frequency, and increase in oxygen and metabolic acidosis needs) were monitored. The following parameters were tested: leucocyte count, platelet count, C-reactive protein test, procalcitonin test, blood culture and peripheral cultures. Morbidity was analysed for both Groups I and II from admission to discharge.

The weights of the infants were monitored from birth to the time they reached 2,500 g. Weighing was carried out daily at a specific time, under the same conditions and using the same
Table I. Weight gain of the extremely low birth weight infants within the first five weeks of life, according to the type of early enteral nutrition given.

\begin{tabular}{lcc}
\hline Early enteral nutrition & \multicolumn{2}{c}{ Average weight gain } \\
\cline { 2 - 3 } & $\begin{array}{c}\text { Per week } \\
(\mathbf{g} / \text { wk) }\end{array}$ & $\begin{array}{c}\text { Per day } \\
(\mathbf{g} / \text { day) }\end{array}$ \\
\hline Human breast milk $(n=18)$ & 120.83 & 17.26 \\
Formula $(n=16)$ & 97.27 & 13.89 \\
\hline
\end{tabular}

scale for both groups. Weight analysis was limited to the first five weeks of life, as all the important weight changes take place in this period in which the infant adapts to postnatal life and achieves full nutrition and weight gain stabilisation. The data collected was processed using descriptive statistics.

\section{RESULTS}

The 34 infants had a birth weight ranging from 850-1,000 $\mathrm{g}$ and a gestational age of $25-33$ weeks. $20(58.8 \%)$ of them were male and $14(41.2 \%)$ were female. $22(64.7 \%)$ infants presented with respiratory distress syndrome with specific symptoms, 27 (79.4\%) had varying degrees of intraventricular haemorrhage, 4 (11.8\%) presented with intraventricular haemorrhage associated with periventricular leucomalacia, and 3 (8.8\%) were diagnosed with ischaemic hypoxic encephalopathy (seen on transfontanellar ultrasonography). Infants with major morbidities were observed to have slower weight gain than those without major morbidities.

The average weight gained per week was $97.27 \mathrm{~g}$ among the infants in Group I (i.e. infants fed with formula) and $120.83 \mathrm{~g}$ among the infants in Group II (i.e. infants fed with human breast milk) (Table I). On average, the infants in Group I gained 19.5\% less weight per day than those in Group II. The difference in the weight gained between the two groups (approximately 20 g) was clinically significant because they were ELBW infants (i.e. $<1,000 \mathrm{~g}$ ).

Length of hospitalisation was monitored according to the type of enteral nutrition that the infants received. The average length of hospitalisation was 106.0 days among the infants in Group I and 97.5 days among the infants in Group II. In the former group, infections are often characterised by septic poisoning. Among the 34 infants, $28(82.4 \%)$ cases of various forms of infections were observed - 18 (64.3\%) cases were early-onset infections (i.e. the infection occurred within the first three days of life), while $10(35.7 \%)$ cases were late-onset infections (Fig. 1).

When the incidence of infection was evaluated according to the type of enteral nutrition the infants received, we found that 16 cases of infection (eight early-onset, eight late-onset) occurred in Group I, and 12 cases of infection (eight early-onset, four late-onset) occurred in Group II. The rates of infection in Group I and Group II were $100.0 \%$ and $66.7 \%$, respectively. Two infants from Group I developed NEC: one infant was treated conservatively, while the other was transferred to the Paediatric Surgery Department.

\section{DISCUSSION}

The initial management of ELBW infants, especially the management of their nutritional needs, is a challenge that neonatal 


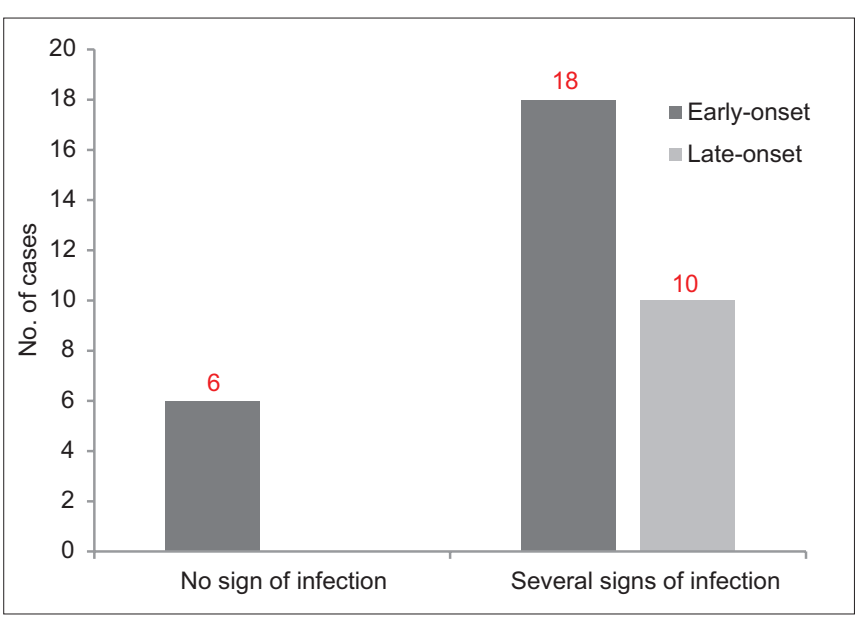

Fig. 1 Bar graph shows the frequency of early- and late-onset infections occurring in the study cohort $(n=34)$.

intensive care units face. Both enteral and parenteral feeding of ELBW infants are typically based on their fluid and caloric needs, which depend on the weight and age of the infant. Such fluid and caloric adjustments occur naturally when the infant is receiving total enteral nutrition with human breast milk. However, in most cases, total enteral nutrition for ELBW infants is only possible after a long period of time; in some cases, it is achieved with formula and not breast milk. ELBW infants are usually placed on a combination of both parenteral and enteral nutrition, with the amount of parenteral nutrition slowly decreasing as the enteral nutrition is slowly increased. This practice is in line with the recommendations by Berseth, ${ }^{(1,3,5)}$ who stated that there should be a gradual increase in the volume rate of enteral nutrition once it has been initiated, and that this gradual increase in volume rate of enteral nutrition should be accompanied by a gradual decrease in the volume rate of parenteral nutrition. This practice was applied to our study cohort.

The presence of comorbidities, which are common among ELBW infants, is signalled by slower weight gain among these infants, as shown in studies conducted by Berseth, and Carver and Barness. ${ }^{(5,13)}$ This was also observed in the present study, in which infants who were fed with human breast milk had a shorter length of hospitalisation as compared to those who were fed with formula (97.5 vs. 106.0 days). The incidence of NEC and neonatal infections with late onset was lower among the human breast milk group. No significant difference was found between the digestive tolerance of the infants who were fed with breast milk and those who were fed with formula.

It should be noted that the present study was limited by its small cohort size $(n=34)$. Due to the small number of infants, the results cannot be generalised and used for the design of clinical guidelines. Further studies involving a greater number of infants should be conducted so that the findings can be useful in the design and establishment of clinical guidelines for the care of ELBW infants.

In conclusion, the need for exclusive parenteral nutrition has been decreasing with the initiation of minimal enteral nutrition. We recommend that neonatal intensive care units promote natural nutrition as the best neonatal metabolic support for ELBW infants.

\section{REFERENCES}

1. Adamkin $\mathrm{DH}$. Issues in the nutritional support of the ventilated baby. Clin Perinatol 1998; 25:79-96

2. Berseth $\mathrm{CL}$, Nordyke C. Enteral nutrients promote postnatal maturation of intestinal motor activity in preterm infants. Am J Physiol 1993:264:G1046-51.

3. Berseth CL. Effect of early feeding on maturation of the preterm infant's small intestine. J Pediatr 1992; 120:947-53.

4. Pereira GR. Nutritional care of the extremely premature infant. Clin Perinatol 1995; 22:61-75

5. Berseth CL. Gastrointestinal motility in the neonate. Clin Perinatol 1996; 23:179-90.

6. Duncan R, Ordonez P, Taguma C. Teaching files: feeding the premature infant. In Neonatology on the Web [online] 2001. Available at: www. neonatology.org/syllabus/feeding.premature.html. Accessed May 15, 2001.

7. Berseth CL. Minimal enteral feedings. Clin Perinatol 1995; 22:195-205.

8. Martinez FE. Uso de nutrição parenteral no período neonatal: princípios e desafios. In: Sociedade Brasileira de Pediatria, organizador. Programa de Atualização em Neonatologia (ciclo 1 módulo 1). Porto Alegre: Artmed/ Panamerican, 2003: 29-58.

9. Jabbar A, Chang WK, Dryden GW, McClave SA. Gut immunology and the differential response to feeding and starvation. Nutr Clin Pract 2003; 18:461-82.

10. Rønnestad A, Abrahamsen TG, Medbø S, Reigstad H, Lossius K, Kaaresen PI, et al. Late-onset septicemia in a Norwegian national cohort of extremely premature infants receiving very early full human milk feeding. Pediatrics 2005; 115:e269-76.

11. Ziegler EE, Thureen PJ, Carlson SJ. Aggressive nutrition of the very low birthweight infant. Clin Perinatol 2002; 29:225-44.

12. Leone CR. Nutrição no recém-nascido com sepse. In: Sociedade Brasileira de Pediatria, organizador. Programa de Atualização em Neonatologia (ciclo 7 módulo 2). Porto Alegre: Artmed/Panamericana, 2003: 117-134.

13. Carver JD, Barness LA. Trophic factors for the gastrointestinal Tract, Clin Perinatol 1996, 23:265-85. 